\title{
NEUROLOGICAL MANIFESTATION ON HOSPITALIZED PATIENT WITH PROBABLE COVID-19 IN SAIFUL ANWAR HOSPITAL INDONESIA (SERIAL CASES)
}

\author{
Badrul Munir ${ }^{1}$, Sri Budhi Rianawati ${ }^{2}$, Shahdevi Nandar Kurniawan ${ }^{2}$, Widodo Mardi Santoso $^{2}$, Eko Arisetijono ${ }^{2}$, Didi \\ Candradikusuma ${ }^{3}$, Sutanto Heri ${ }^{3}$, Iin Noor Chozin ${ }^{4}$, Pratiwi Suryanti Dwi ${ }^{4}$, Tri Wahju Astuti ${ }^{4}$, Syaifullah Asmiragani $^{5}$ \\ Correspondence: Badroel2007@ub.ac.id

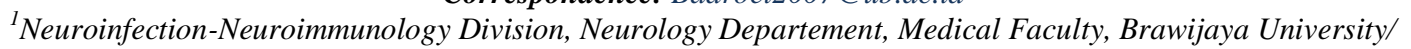 \\ Covid-19 Task Force Saiful Anwar Hospital, Malang, Indonesia \\ ${ }^{2}$ Neurology Departement, Medical Faculty, Brawijaya University, Malang, Indonesia \\ ${ }^{3}$ Tropical Infection Division, Medical Faculty Brawijaya University/Covid-19 Task Force Saiful Anwar Hospital, Malang, Indonesia \\ ${ }^{4}$ Pulmonology Department Medical Faculty Brawijaya University/Covid-19 task force Saiful Anwar Hospital, Malang, Indonesia \\ ${ }^{5}$ Orthopedist, Medical Faculty Brawijaya University/Head of Covid-19 Task Force Saiful Anwar Hospital, Malang, Indonesia
}

\section{Article History: \\ Received: June 5, 2020 \\ Accepted: June 15, 2020 \\ Published: July 1, 2020}

\section{Cite this as:}

Munir B, Rianawati SR,

Kurniawan SN, Santoso WM,

Arisetijono E, Candradikusuma

D, Heri S, Chozin IN, Dwi PS,

Astuti TW, Asmiragani S.

Neurological manifestation on

hospitalized patient with probable

covid-19 in saiful anwar hospital indonesia (serial cases). Malang

Neurology Journal; 2020.6:51-

55.

http://dx.doi.org/10.21776/ub.mnj .2020.006.02.1

\begin{abstract}
Background: Coronavirus disease 2019 (Covid-19) caused by SARS-Cov-2 had been spreading worldwide including Indonesia, but In Indonesia, it was difficult to diagnose confirmation Covid-19 by Polymerase Change Reaction (PCR). and in clinical practice to treat covid-19, Indonesia Health Ministry use term probable covid-19 for patients under observation Covid-19, but study for neurological manifestation on probable Covid-19 is very lacking.

Objective: Knowing the neurological manifestation of patient probable Covid-19.

Methods: Observational study from Covid-19 medical record and neuro-Covid-19 medical record of all probable Covid-19 hospitalized in the isolation ward of Saiful Anwar Hospital (RSSA) Malang since March-May, 2020 (3 months). Probable Covid-19 criteria were given by Covid-19 task force team based on Health Minister Indonesia criteria. This criterion was based on clinical manifestation (severe upper respiratory symptoms, or mild-severe pneumonia), radiologic examination (lung infiltrate and or chest CT scan), and laboratory examination. Neurological manifestation is based on clinical symptom appeared while patient hospitalized, supported with radiologic and laboratory data which was supervised by neurologist or neurologist resident. All data was inputted to neuro-Covid-19 e-registry on the smartphone application, sent periodically to the data center in Neurology Department RSSA Malang.

Results: A hundred and fourteen probable Covid-19 was registered with mean age 34,5 year old and mostly at the young age of 20-50 years (38\%). The neurological manifestation was observed in 4 patients (3,4\% from all possible Covid-19 patients) with thrombotic stroke as the most common manifestation $(50 \%)$, followed by myelum tumor $(25 \%)$ and hypoxia encephalopathy $(25 \%)$. Stroke patients in probable Covid-19 had old age (72 and 82 years) accompanied by several comorbid such as hypertension, DM and CVA history and Covid symptoms of cough, shortness of breath and fever. Hemiparesis was found improved with aspirin and neurotropic therapy. Patient with myelum tumor had a covid symptom of shortness of breath, cough, and fever with UMN tetraparesis. Previous MRI examination results suspected meningioma. The patient died while hospitalized. Patients with hypoxia encephalopathy admitted with decreased consciousness due to shortness of breath with acidosis respiratory symptoms $(\mathrm{pH} \mathrm{7,22})$. This condition improved after oxygenation without focal neurologic deficit.

Conclusion: Neurological manifestation in probable Covid-19 was 3,4\% of all patients. The most common diagnosis was a thrombotic stroke, followed by myelum tumor, hypoxia encephalopathy, and mortality rate is $25 \%$.
\end{abstract}

Keywords: Covid-19, manifestation, neurology, stroke, probable

\section{Introduction}

Coronavirus disease-2019 (Covid-19) caused by the SARSCoV-2 virus initially was found in Wuhan Province, China, and then spread worldwide, including Indonesia. Yet, Covid-19 diagnosis in Indonesia was slow and difficult. This was due to the limited number of gold standard examination Polymerase Chain Reaction (PCR) for Covid-
19, attributed to lack of resources and infrastructure. For Covid-19 management, Indonesia government coined several terms for diagnosis, the terms are: person under observation related suspect covid, patient under observation related probable covid, and confirmed case of Covid-19.

Suspect Covid-19 had mild symptoms such as fever, upper respiratory symptoms, and had close contact with positive Covid-19 patients or travel history from the endemic region 
(Depkes RI 2020). Probable Covid-19 is people from the local transmission area or had contact with positive Covid19 patients who had a severe upper respiratory symptom or mild to severe pneumonia. In this case, people with symptoms need to be attended in the Emergency Hospital or Referral Hospital. ${ }^{1}$

Clinical manifestation of Covid-19 was; fever 61,7\%, cough $50 \%$, shortness of breath, and anorexia $31,8 \%$. Meanwhile, neurological manifestation was found in $36,4 \%$ of all Covid-19 patients. Neurological symptoms were categorized into 3 types; muscle disorder $(10,7 \%)$, central nervous system disorder (24\%), and peripheral nervous system disorder $(8,9 \%)$. $^{2,3}$

The manifestation of central nervous system disorder ranged from cephalgia, decreased consciousness, stroke, ataxia, and seizure. Peripheral nervous system disorder includes disorder of tongue $(5,6 \%)$, nose $(5.6 \%)$, vision, and pain. ${ }^{2}$

The neurological damage mechanism in Covid-19 through several pathways. The neuronal pathways could be explained through viral transport from the olfactory nerve through the olfactory bulb which is the canal between nasal epithelial and central nervous system. At initial infection, this pathway was prone to hypoxia damage. Hypoxemia condition in SARS-CoV-2 infection causes hypoxia in CNS, increasing anaerobic metabolism which in turn triggers cerebral vasodilation, brain tissue edema, and subsequent brain ischemic. Inflammatory pathways in severe infection would cause Systemic Inflammatory Response Syndrome (SIRS) where there were cytokines storms due to SARS-CoV-2 infection, activating IL-6, IL-2, IL-15, TNF- $a$ in glial cells. ACE2 pathway could be explained by the interaction of SARS-CoV-2 protein spikes with ACE2 expressed in endothelial, which cause blood brain barrier impairment allowing the virus to enter the CNS (ACE-2). ${ }^{4,5}$

Based on data in the Health Ministry of Indonesia until end of May 2020 showed 47.714 were suspected Covid-19, 12.832 were probable covid-19, and 25.773 were positive confirmed Covid-19 . Data from East Java province until the end of May 2020; 24.616 were suspected Covid-19, 6.609 were probable and 4.848 were positive confirmed Covid-19 with total mortality of $412(8,4 \%){ }^{1}$

Yet, there was no reported neurological manifestation reported from probable Covid-19, especially in Indonesia. Therefore this study needs to be done. Aim of this study was to understand neurological manifestation in probable Covid-19 admitted in RSSA Malang and their condition at discharge.

\section{Methods}

This was an observational study by analyzing patient medical records and special neuro-Covid-19 medical records of patients in Covid-19 from isolation ward RSSA Malang March-May 2020 (3 months).

Probable criteria were decided by Covid-19 Task Force Saiful Anwar Hospital based on Health Minister criteria. Covid-19 Task Force team consisted of multidisciplinary specialists (pulmonologist, internist, clinical pathology, microbiology, radiology). Patients criteria were based on their clinical manifestation, radiologic examination, laboratory examination at admission to emergency ward. ${ }^{1}$

Based on health Minister Indonesia, Criteria of possible Covid-19 include: fever/history of fever, cough/pain of through/flue syndrome, dyspnea (clinical picture and or radiology examination shows mild-severe pneumonia) add one risk factor at least 14 days before onset symptoms as traveling or live in local transmission or history close contact with patient confirm covid $-19 .^{1}$

A neurological examination was done by a neurologist. The neurological examination included the level of conscientious, meningeal sign, cranial nerve, motorysensory, physiologic and pathologic reflexes, and specific examination as needed (MMSE, Siriraj stroke score, etc).

Stroke diagnosis was based on WHO consensus with criteria: acute neurological deficit due to brain circulation impairment supported by head CT Scan and or MRI examination. Encephalopathy was based on the clinical symptom of decreased consciousness, with metabolic impairment supported by head CT scan and supported by laboratory examination. Myelum tumor diagnosed by the clinical symptom of UMN tetraparesis/paraparesis supported by X-ray, MRI, and lumbal puncture.

Data collected from patients were clinical, laboratory, and radiologic data. These data were entered into Covid-19 eregistry (in smartphone application) and analyzed periodically for data validity. This study was approved by the Ethics Board of Saiful Anwar Hospital Malang.

\section{Results}

One hundred fifty three patients admitted in isolation room Saiful Anwar Hospital for 3 months (March-May, 2020). Ratio male: female ( $52 \%$ vs $48 \%$ ) with most patients aged above 50 years old. There were 114 patients with probable Covid-19 and 39 patients were confirmed Covid-19.

Of all patients with probable covid-19 has consulted to neurology department, there were 4 patients $(3,5 \%$ of all possible Covid-19). Data based patient with neurological manifestation in possible Covid-19; a range of age 45-82, female: male $(25 \%$ vs $75 \%)$, with diagnosis stroke infarction (2 patients), myelum tumor (1 patient), and hypoxic encephalopathy ( 1 patien) with a mortality rate of $25 \%$

\section{Case 1}

Male, 87 years old, admitted to the hospital with shortness of breath and cough since 7 days ago and the patient had sudden left side weakness 2 days after admission in Covid19 isolation ward, history of hypertension, DM, and stroke.

Physical examination at admission was as follows; blood pressure 130/80 $\mathrm{mmHg}$, pulse 67 byte/minutes, respiratory rate: 20 times/minutes, axillary temperature $36,5^{\circ} \mathrm{C}$. Neurological examination GCS E4 V5 M6 (GCS 15), paresis of left facial and lingual nerve UMN type, Motoric strength of right side was 5 and left side was 3, normal physiological reflexes, negative pathological reflexes, Sirijac Stroke Scale (SSS) was -2 (indicating stroke infarction), laboratory examination results were random blood glucose $128 \mathrm{mg} / \mathrm{dl}$, HBA1c 5,3\%, troponin 0,10, CKMB 18. Chest Xray examination found infiltrate in both 
lung, brain CT scan found infarction in the right internal capsule, the patient was admitted for 10 days in an isolation ward with a diagnosis of CVA thrombotic third attack with PDP severe Covid-19, the patient was discharged alive with no improvement in motoric strength and PCR Covid-19 was on progress.

\section{Case 2}

Male 72 years old, admitted to hospital with a chief complaint of sudden left side weakness 4 days before admission. The patient also complained of shortness of breath since 1 day before admission. He had a history of hypertension, stroke, heart disease. Physical examination at admission was as follows; blood pressure 130/80 $\mathrm{mmHg}$, pulse 84 byte/minutes, respiratory rate 20 times/minute, axillary temperature $37^{\circ} \mathrm{C}$. GCS E4M5V6 (15) paresis of left facial nerve UMN type, left side motoric strength was 5 and the right side was 4, normal physiological reflexes, Positive pathological Babinski reflex. At discharge, cranial nerve paresis was disappeared with improved motoric strength (4+). The length of stay was 14 days in the isolation ward. The patient was discharged alive and the PCR Covid-19 result was not available yet.

Table 1. Baseline hospitalized patients related Covid-19

$$
\text { ( } \Sigma: 153 \text { patients) }
$$

\begin{tabular}{ll}
\hline \multicolumn{1}{c}{ Criteria } & \\
\hline Gender (male:female) $(\%)$ & $52 \%: 48 \%$ \\
Mean Age (year old) & $35(0-82)$ \\
Distribution based on age (years) & \\
$0-10$ & $28(18 \%)$ \\
$11-20$ & $4(2,6 \%)$ \\
$21-50$ & $58(38 \%)$ \\
$51-60$ & $34(22 \%)$ \\
$61-70$ & $20(13 \%)$ \\
$71-80$ & $7(4,6 \%)$ \\
Positive confirmed Covid-19 & $39(26 \%)$ \\
\hline
\end{tabular}

\section{Case 3}

Female 45 years old admitted to hospital gradual weakness in all four extremities for 3 weeks progressively before admission. Other symptoms were numbness in all extremities for 3 weeks before admission, shortness of breath since 5 days before admission. The patient had cervical cancer since 2014. The patient had a history of hysterectomy and radiotherapy.

Physical examination at admission found that the patient appeared weak, blood pressure $90 / 60 \mathrm{mmHg}$, respiratory rate 26 times/minutes, axillary temp $36,6^{\circ} \mathrm{C}$. Neurologic status: GCS E4M5V6, MMSE: 20, the cranial nerve within a normal range, tetraparesis on motoric examination with motoric strength of upper right 4, upper left 5, lower right 0 , lower left 5.

Sensory examination was as follows; Protopathic: hypoesthesia vertebrae Th3-4 and Proprioceptive: impairment of inferior extremity, increased physiological reflexes, and positive pathologic reflexes. Laboratory result was anemic with $\mathrm{Hb}$ : 8.20, leukocytosis (16.050) and decreased lymphocyte, Neutrophil: 87,2, Lymphocyte: 5.0, Monocyte: 7.2, Ureum: 79.6, Creatinine:2.23, Natrium 121, Kalium 4.84, Chloride 96. Chest radiography found infiltrate in both lungs. The patient had a working diagnosis of tetraparesis UMN type due to myelum tumor of extremities at segment vertebrae Th 1-2 due to suspected metastatic process with differential diagnosis of compression fracture with pneumonia due to moderate possible Covid-19.

The patient was admitted in an isolation ward for 3 days, supervised by a multidisciplinary team. The patient had the worsening condition on the second day, with worse shortness of breath. The patient was died probably due to sepsis.

\section{Case 4}

Female, 71 years old with a chief complaint of gradual decrease of consciousness after the patient had shortness of breath. Shortness of breath was felt 2 days before admission. After oxygenation in the emergency ward, there was an improvement in saturation from $40 \%$ to $99 \%$. There were fever and cough, the patient had no focal neurological deficit either motoric, sensory, or autonomic. History of disease: heart disease found 6 months before admission.

Physical examination at admission was as follow; blood pressure 126/76 $\mathrm{mmHg}$, pulse 100 times/minute, respiratory rate 22 times/minutes, axillary temperature $36^{\circ} \mathrm{C}$, GCS from E2M2V5 (8) improved to E4M5V6 (15) after oxygenation treatment, Meningeal sign (-) cranial nerve: isochoric pupil $3 \mathrm{~mm} / 3 \mathrm{~mm}$, light reflex $+/+$, corneal reflex $+/+$, normal motoric and sensory, normal physiological reflexes, negative pathological reflexes.

Laboratory results: Blood Gas Analysis (BGA): $\mathrm{PH}$ 7.27/pCO :36.8/ $\quad \mathrm{HCO}_{3} \quad 128.2 / 17 / \mathrm{BE} \quad-10.1 / 98.3 \%$, haemoglobin: 11,4 , leukocyte $21780 /$ thrombocyte 288.000 , Diff count: 0,0/0,1/89,7/4,8/5,4, LED: 45, Natrium 137, kalium 4,37, chloride 113, SGOT 91, SGPT39, Alb: 3,7, GDS: 171, Ureum/Creatinin: 75,1/1,9, CRP: 1,1, Procalc: 6,78. Thorax examination: Cardiomegaly with central infiltrate. Patient was admitted with probable COVID-19 post hypoxia encephalopathy.

\section{Discussion}

Neurological manifestation in probable Covid-19 in 4 patients $(3,5 \%$ of all probable Covid-19) it was different from a previous study that shows neurological manifestation in Covid-19 has 36,4\% from covid-19 patient. This differences might be due to this study in probable Covid-19 and not on confirmed positive Covid19 , it was caused unavailability of PCR covid-19 result while hospitalized. ${ }^{2}$

The most common neurological manifestation in probable Covid-19 was stroke infarction, followed by myelum tumor and encephalopathy. Patients usually had old age (64-85 yo). This was in line with the previous study shows stroke manifestation was around $5-5,7 \%$ of all Covid-19 cases. In these elderly patients, stroke infarction was the most common manifestation, in our study infark stroke 2 patients $(50 \%$ of neurologically manifested in probabe covid-19). This supported a previous study that showed that CVA was the most common manifestation in Covid19 cases in the central nervous system. ${ }^{6}$

Clinical symptoms of Covid-19 were shortness of breath $(100 \%)$ fever $(100 \%)$ and cough $(50 \%)$. An additional result of infiltrate in both lung and positive rapid tests caused the patient to be diagnosed with probable covid-19. This was in line with a previous study where several 
clinical symptoms were fever $(61,7 \%)$, cough $(50 \%)$, and shortness of breath. In a previous study, the patient had undergone additional examination such as a radiologic examination (chest CT scan). In our study, the patient had not had a chest CT scan and PCR Covid-19 test. ${ }^{2}$

In this study, patients' ages were relatively old, accompanied by other risk factors such as hypertension, DM, and heart disease. This was following Mao ling study which stated that there were a correlation between stroke event with the number of risk factors patient had. The more severe Covid-19 was, the more stroke incident happened.
Cerebral infarction mechanisms could be related to hypoxemia. Hypoxemia condition in SARS-CoV-2 infection causes hypoxia in CNS, increasing anaerobic metabolism which in turn triggers cerebral vasodilation, brain tissue edema, and subsequent brain ischemic. Inflammatory pathway in severe infection would cause Systemic Inflammatory Response Syndrome (SIRS) where there were cytokines storms due to SARS-CoV-2 infection, activating IL-6, IL-2, IL-15, TNF- $a$ in glial cells. ACE2 pathway could be explained by the interaction of SARS-CoV-2 protein spikes with ACE2 expressed in endothelial, which cause blood brain barrier impairment allowing the virus to enter the CNS. ${ }^{2,4,5,7}$

Table 2. Baseline data of CVAThrombotic with probable Covid-19

\begin{tabular}{|c|c|c|}
\hline Criteria & Case 1 & Case 2 \\
\hline Age (years) & 87 & 72 \\
\hline $\operatorname{sex}$ & Male & Male \\
\hline \multicolumn{3}{|l|}{ Covid-19 symptoms : } \\
\hline Fever & $+(7$ days $)$ & $+(5$ days $)$ \\
\hline Cough & $+(7$ days $)$ & - \\
\hline Shortness of breath & $+(7$ days $)$ & $+(5$ days $)$ \\
\hline $\begin{array}{l}\text { History of contact with positive } \\
\text { Covid-19 }\end{array}$ & + & + \\
\hline History of past illness & Hypertension, DM, CVA & Hypertension, heart disease, CVA \\
\hline Blood pressure $(\mathrm{mmHg})$ & $130 / 80$ & $180 / 80$ \\
\hline Initial symptom at admission & Acute left hemiparesis & Acute left hemiparesis \\
\hline \multicolumn{3}{|l|}{ Neurological Examination } \\
\hline GCS (EMV) & 456 & 456 \\
\hline Meningeal sign & - & - \\
\hline Paresis of cranial nerve & $\begin{array}{c}\text { Paresis of left cranial nerve } \\
\text { VII and XII }\end{array}$ & $\begin{array}{c}\text { Paresis of left cranial nerve VII } \\
\text { and XII }\end{array}$ \\
\hline Motoric power & 53 & 54 \\
\hline & 53 & 54 \\
\hline Sensory & Left hemihypoesthesia & Normal \\
\hline Siriraj stroke stroke (SSS) & -2 & -1 \\
\hline Head CT Scan & infarcted right internal capsul & No lesion seen \\
\hline$X$ ray thorac & Infiltrate in both side of lungs & infiltrat parenchemal lungs \\
\hline Covid-19 rapid test antibody & + (reactive) & + (reactive) \\
\hline Random blood glucose & 128 & 121 \\
\hline HBA1c & 5,3 & NA \\
\hline Haemoglobin (dl & 11,7 & 10,6 \\
\hline Leukocyte & 7960 & 3480 \\
\hline Neutrifil & $74,4 \%$ & $87,3 \%$ \\
\hline Limfosit & $0,11 \times 10^{3}$ & $0,27 \times 10^{3}$ \\
\hline Monosit & $0,9 \times 10^{3}$ & $0,14 \times 10^{3}$ \\
\hline Natrium & 133 & 135 \\
\hline Kalium & 3,42 & 3,08 \\
\hline SGPT & 28 & 88 \\
\hline SGOT & 17 & 268 \\
\hline Albumin & NA & NA \\
\hline Ureum & 23,5 & 81,6 \\
\hline Creatinin & 1,12 & 2,13 \\
\hline Troponin & 0,10 & NA \\
\hline CKMB & 18 & NA \\
\hline LDH : & NA & 1539 \\
\hline Final Diagnosis & $\begin{array}{l}\text { CVA thrombotic } 3^{\text {th }} \text { attack } \\
\text { and severe probable Covid-19 }\end{array}$ & $\begin{array}{l}\text { CVA thrombotic } 2^{\text {nd }} \text { attack } \\
\text { +andmoderate probable Covid-19 }\end{array}$ \\
\hline Discharge from hospital & alive & alive \\
\hline Length of stay (days) & 10 & 14 \\
\hline
\end{tabular}

But there were possibilities where stroke had no direct correlation but was comorbid of Covid-19 since in these patients there was a classical factor of stroke infarction such as old age, hypertension, DM and dyslipidemia related to microvascular atherosclerosis process causing thrombus and neuron damage with the various mechanism ${ }^{6}$

Both of stroke infarction patients were treated with aspirin and neurotropic. Although there were extracranial improvement and discharged in a much better although not 
completely healed, this result was still in line with previous case report where stroke in Covid-19 patient had good prognosis.

Another manifestation was myelum tumor due to the metastatic process. This case was probably only comorbid with probable Covid-19 since tumor manifestation had appeared before Covid-19 manifestation such as shortness of breath. Patients in this case also had other complications such as renal impairment, which could indicate the malignancy process. There was a scarce study regarding CNS tumor correlation with Covid-19, but one study showed Covid-19 with tumor comorbid as much as $24,6 \%$ and Covid-19 patient with a history of tumor of only $0,9 \% .^{8}$

Myelum tumor diagnosis was established based on the clinical manifestation of progressive tetraparesis UMN supported with MRI examination before admission, where there was mass at a cervical level similar to meningioma, the most common type of myelum other than metastasis. A previous study stated that primary myelum tumor incidence were meningiomas $(29 \%)$, nerve sheath tumors (24\%), and ependymomas $(23 \%){ }^{9}$

The patient was admitted in severe condition with infection sign, and worsen while hospitalized. This was shown by increased leukocyte, decreased renal function, and sepsis sign. The cause of death, whether it was correlated to Covid-19 or not, was not clearly understood. But based on the sign and symptoms, this could be due to sepsis due to spinal tumor complications.

In the fourth case, females presented with a decreased of consciousness after shortness of breath. History of fever and cough arouse suspicion of Covid-19. Neurological examination at admission had GCS E2 V2 M4 without a neurological deficit. Blood gas analysis showed decreased $\mathrm{pH} ; 7,22$ with decreased pCO2 level, indicating acidosis. respiratory. The patient should underwent a head CT scan, but the patient had recovered before the test could be done. Decreased of consciousness was one of Covid-19 manifestation in neurology, the previous study showed that 2,4-14,8\% Covid-19 patient had decreased of consciousness and correlated with the severity of disease Covid-19. ${ }^{2}$

The cause of decreased consciousness in this case probably was attributed to hypoxia decreasing blood circulated to the brain and lowering brain metabolism. This caused global ischemic and coma were metabolic. The patient had recovered after oxygenation treatment using NRBM $100 \%$ without a neurological deficit sequel. Neurologic examination showed that there was no stroke sign due to focal disturbance of blood circulation. ${ }^{10,11}$

Limited this study are case series design on possible covid-19 and not on confirmed covid-19. it were caused by unavailability PCR testing when patient hospitaized, this study has limited samples too, Further study with larger samples and faster PCR examination was needed to validate manifestation neurological in covid-19.

\section{Conclusion}

Neurological manifestation of probable Covid-19 was found in $3,4 \%$, stroke infarction was the most common manifestation, followed by myelum tumor and hypoxia encephalopathy, and the mortality rate is $25 \%$.

\section{Conflict of Interest}

No conflict of interest in this study.

\section{Acknowledgement}

The author thanks to Covid-19 Task Force Saiful Anwar General Hospital, Malang, Indonesia.

\section{References}

1. Kemenkes RI. Pedoman Pencegahan dan Pengendalian Coronavirus Disease (COVID-19), Germas; 2020. pp. 0-115

2. Mao, L. et al. Neurologic manifestations of hospitalized patients with coronavirus disease 2019 in Wuhan, China. JAMA Neurology; 2020.

DOI: $10.1001 /$ jamaneurol.2020.1127

3. Sedaghat, Z. and Karimi, N. Guillain Barre syndrome associated with COVID-19 infection: A case report, Journal of Clinical Neuroscience. Elsevier Ltd, (xxxx); 2020. pp. 10-12.

DOI: $10.1016 /$ j.jocn.2020.04.062.

4. Baig, A. M. et al. Evidence of the COVID-19 virus targeting the CNS: Tissue distribution, host-virus interaction, and proposed neurotropic mechanisms'. ACS Chemical Neuroscience; 2020. 11(7):995-998. DOI: $10.1021 /$ acschemneuro.0c00122.

5. Avula, A. et al. COVID-19 presenting as stroke, brain, behavior, and immunity. Elsevier; 2020. April, pp. 01. DOI: $10.1016 /$ j.bbi.2020.04.077

6. Wilkinson, R. J. et al. Tuberculous meningitis', nature reviews neurology. Nature Publishing Group; 2017. 13(10):581-598. DOI: 10.1038/nrneurol.2017.120

7. Guo, Y. et al. The origin, transmission, and clinical therapies on coronavirus disease 2019 (COVID-19) outbreak - an update on the status'. Military Medical Research; 2020. pp. 1-10. Avalaible from: https://mmrjournal.biomedcentral.com/articles/10.118 6/s40779-020-00240-0

8. Moujaess E, Kourie HR, and Ghosn M. Cancer patients, and research during COVID-19 pandemic: A systematic review of current evidence. Critical Reviews in Oncology/Hematology; 2020. DOI: 10.1016/j.critrevonc.2020.102972.

9. Schellinger KA et al. Descriptive epidemiology of primary spinal cord tumors. Journal of NeuroOncology; 2008. 173-179. DOI: 10.1007/s11060-0079507-z

10. Munir. Relationship between the random blood glucose levels during admission at emergency room with clinical output in acute ischemic stroke patients; 2015. 52-60.

DOI: http://dx.doi.org/10.21776/ub.mnj.2015.001.02.2

11. Allen KA and Brandon DH. Hypoxic ischemic encephalopathy: Pathophysiology and experimental treatments. Newborn and Infant Nursing Reviews; 2011. 11(3). 125-133.

DOI: 10.1053/j.nainr.2011.07.00 